\title{
Low FT3: a possible marker of frailty in the elderly
}

\author{
Aldo Bertoli' \\ Alessia Valentini' \\ Maria Assunta Cianfarani \\ Elena Gasbarra ${ }^{2}$ \\ Umberto Tarantino ${ }^{2}$ \\ Massimo Federici \\ 'Department of Systems Medicine, \\ University of Rome "Tor Vergata", \\ Rome, Italy; ${ }^{2}$ Department of \\ Orthopaedics and Traumatology, \\ University of Rome "Tor Vergata", \\ Rome, Italy
}

This article was published in the following Dove Press journal:

Clinical Interventions in Aging

10 February 2017

Number of times this article has been viewed

Introduction: Frailty is associated with a functional decline of multiple physiological systems, of which they may be a cause or consequence. The objective of the study was to evaluate the prevalence of thyroid hormone modifications in elderly frail subjects and its relationship with frailty.

Study population and methods: An observational study was carried out at the University Hospital "Tor Vergata" in Rome among ambulatory and hospitalized patients. The study population consisted of 112 elderly subjects: 62 were hospitalized following hip fracture and 50 control subjects were outpatients. Participating patients received a multidimensional geriatric evaluation. The Survey of Health, Ageing and Retirement in Europe Frailty Instrument (SHARE-FI) was used to assess the degree of frailty. Thyroid stimulating hormone (TSH), free triiodothyronine (FT3), and free thyroxine (FT4) were measured to evaluate thyroid status.

Results: FT3, but not FT4, was significantly correlated with Frailty score, both in patients with hip fracture and in patients from the control group. In the entire study population, FT3 under normal limits is effective in discriminating frail/prefrail subjects from nonfrail subjects.

Discussion: The reduction in serum concentrations of FT3 is a clear manifestation of stress associated with fractures. Numerous preexisting factors, such as the fracture patients' nutritional status, sarcopenia, disability and comorbidities, which characterize the condition of frailty and influence its pathogenesis, are strongly correlated with FT3 values, suggesting the existence of latent nonthyroidal illness syndrome (NTIS).

Conclusion: We conclude that measuring FT3 can be a useful laboratory parameter in clinical assessment, which can play an important role in identifying vulnerable elderly subjects and in quantifying the condition of frailty.

Keywords: FT3, frailty, NTIS, hip fracture, aging

\section{Introduction}

Most industrialized countries are experiencing progressive aging of their populations. ${ }^{1}$ In Italy, the percentage of people older than 65 years is currently equal to $21.7 \%$ and is projected to rise further, exceeding $33 \%$ by $2050 .^{2}$ Aging is associated with a progressive increase in the prevalence of frailty. ${ }^{3}$

Frailty is a condition characterized by reduced resistance to stressful events, stemming from a functional decline of multiple physiological systems. This results in an alteration of the homeostatic mechanisms of the body and helps to increase the risk of adverse events, such as hospitalization, institutionalization and death. ${ }^{3}$

To date, there is no universally accepted definition of frailty. Several models have been proposed to identify and measure it, ${ }^{4,5}$ including some based on clinical criteria ${ }^{6}$ as proposed by Fried et $\mathrm{al}^{3}$ and others based on laboratory tests. ${ }^{7,8}$

The progressive decline in the function of various organs and systems in the elderly contributes to a reduction in the homeostatic capacity of the body, leading to frailty. We argue that changes in the endocrine system can play a decisive role in this process.
Correspondence: Aldo Bertoli Department of Systems Medicine, University of Rome "Tor Vergata", Via Montpellier 8I, Rome 00133, Italy

Tel +390620903618

Fax +3906 20904955

Email aldo.bertoli@uniroma2.it $\mathrm{BY}$
hereby accept the Terms. Non-commercial uses of the work are permitted without any further permission from Dove Medical Press Limited, provided the work is properly attributed. For permission for commercial use of this work, please see paragraphs 4.2 and 5 of our Terms (https://www.dovepress.com/terms.php). 
Physiologically, the endocrine system intervenes in regulating energy metabolism and stress response. In the elderly, the decline in endocrine function is particularly evident in the presence of stressful events. ${ }^{9}$ Endocrine changes that occur with age involve all the glands and, although the relationship between frailty, declining age-related concentrations of androgens and growth hormone $(\mathrm{GH})$ on the one hand ${ }^{10}$ and adrenal function on the other is well known, the role of thyroid hormones is still under debate.

Thyroid abnormalities have been suggested as constituting a possible predisposing factor of frailty in elderly patients with chronic kidney disease (CKD) by Abdel-Rahman et al. ${ }^{11}$ However, in an exhaustive review, Morley et al ${ }^{12}$ conclude that there is little evidence that thyroid hormone plays a role in the pathogenesis of frailty. Recently, FernándezGarrido et $\mathrm{al}^{13}$ assessed the clinical features of prefrail older individuals and emerging biomarkers and argued that "the identification of biomarkers associated with prefrailty state would be very helpful".

With advancing age, we observe a progressive reduction in serum triiodothyronine (T3) concentrations, while the levels of thyroxine (T4) and thyroid stimulating hormone (TSH) do not change significantly. ${ }^{9}$ These values are suggestive of nonthyroidal illness syndrome (NTIS). This article evaluates the prevalence of NTIS and describes the relationship between NTIS and frailty.

\section{Study population and methods}

The data presented in this article are derived from an observational study conducted at the Polyclinic Tor Vergata in Rome; the study objective was to evaluate the main indicators of frailty and their relationship with changes in the endocrine system in patients aged 65 years or above, with or without low-energy fractures.

The study population included 112 elderly subjects referred to the Polyclinic Tor Vergata. Of these, 62 were enrolled among the patients hospitalized in the Orthopedic Department after a hip fracture and 50 control subjects were enrolled among outpatients evaluated at the Department of Medicine (Clinical Program on Atherosclerosis). All investigations were carried out according to the Declaration of Helsinki, as modified in 2000, including obtaining written informed consent from all participants, and the study protocol was approved by the ethical committee of the Polyclinic Tor Vergata.

Inclusion criteria comprised an age of $\geq 65$ years in both groups and current hip fracture in hospitalized patients. Exclusion criteria included the presence of a malignancy or a history of previous cancer.
Each patient's sex and age were recorded, anthropometric parameters were measured (weight and height), and the body mass index (BMI) was calculated. The medical history of each patient was recorded, including drugs taken chronically. All study participants underwent physical examination and blood sampling for laboratory assays (blood count, creatinine, glucose, albumin, serum cortisol, TSH, free T3 [FT3], free T4 [FT4], high-sensitivity C-reactive protein [hs-CRP], interleukin-6 [IL-6] and tumor necrosis factor- $\alpha[$ TNF- $\alpha])$. The blood count and glucose concentration were assessed by routine laboratory tests (Sysmex XE 2100; Dasit), while creatinine and albumin were measured by homogeneous-phase chemiluminescent enzyme immunoassay (Dimension VISTA 1500; Siemens). TSH (reference values: $0.35-4.5 \mu \mathrm{IU} / \mathrm{mL}$ ), FT3 (reference values: 2.3-4.2 pg/mL), FT4 (reference values: $0.8-1.75 \mathrm{ng} / \mathrm{dL}$ ) and plasma cortisol were measured by chemiluminescence assay (ADVIA Centaur XP; Siemens); hs-CRP was measured using nephelometric method (Dimension VISTA 1500). The levels of IL- 6 and TNF- $\alpha$ were determined with enzyme immunoassay (DRG Diagnostics).

Participating patients received a multidimensional geriatric evaluation comprising the following scales: Activities of Daily Living (ADL) ${ }^{14}$ and Instrumental Activities of Daily Living (IADL); ${ }^{15}$ Mini-Mental State Examination (MMSE) $;{ }^{16}$ Geriatric Depression Scale (GDS); ${ }^{17}$ and Mini Nutritional Assessment (MNA). ${ }^{18}$ Comorbidities were assessed using the Cumulative Illness Rating Scale for Geriatrics (CIRS-G), from which we obtained the CIRS severity (CIRS-S), CIRS comorbidity index (CIRS-CI), ${ }^{19}$ and Charlson comorbidity index (CCI) ${ }^{20}$ In patients able to collaborate, muscle strength was measured through handgrip dynamometry, and the degree of frailty was subsequently calculated using the Survey of Health, Ageing and Retirement in Europe Frailty Instrument (SHARE-FI). ${ }^{6}$ Patients enrolled in the study who were able to be moved without difficulty underwent dual-energy X-ray absorptiometry (DEXA) for the evaluation of lumbar and femoral bone mineral density (BMD), lumbar and femoral $\mathrm{T}$-Score and Z-Score as well as the body composition.

\section{Statistical analysis}

The Kolmogorov-Smirnov test was used to assess normal distribution variables. Student's $t$-test and analysis of variance (ANOVA) were used to compare continuous variables. Pearson's linear regression was used to correlate continuous variables. Nonparametric correlations were evaluated using Spearman's rank correlation, as indicated. One-way ANOVA and simple linear correlation were used to assess the relationship between continuous variables. 
Logistic regression was used to assess the relative influence of independent variables on FT3 and the Frailty score.

Analysis of covariance (ANCOVA) was used to assess the weight of the fracture on the relationship between FT3 and the Frailty score. An analysis of the receiver operating characteristic (ROC) curve and the area under the curve (AUC) was undertaken to assess the accuracy of FT3 in the distinction between frail and nonfrail subjects. All data are presented as mean \pm standard deviation (SD). Values of $P<0.05$ were considered significant. Statistical analysis was performed using Stat View 5 (SAS Institute, Cary, NC, USA). Graphs were designed using GraphPad Prism 5 (GraphPad Software Inc, San Diego, CA, USA).

\section{Results}

The study population consisted of 112 patients, of both sexes, aged between 65 and 98 years, whose anthropometric characteristics and features related to body composition are reported in Table 1. The data relating to comorbidity, frailty and multidimensional assessment are reported in Table 1.

Compared to subjects in the control group, patients with fractures had significantly lower average values of hemoglobin and albumin, as well as significantly greater average values of white blood cells (WBCs) and inflammatory markers (Table 2).
Patients with fractures had mean values of FT3 significantly lower than those observed in patients in the control group. The two groups did not differ in terms of mean values of TSH and FT4 (Table 2).

About $42.3 \%$ of the study participants had low levels of FT3: the prevalence of this condition was higher in the group with fractures compared with the control subjects $(40.2 \%$ vs $2.1 \%, P<0.001)$.

Unlike FT4, FT3 was correlated with nutritional status (MNA), the degree of disability (ADL and IADL), comorbidities (CIRS-G) and muscle strength (handgrip), as shown in Table 3.

A linear regression analysis (Figure 1A and B) showed the absence of a statistically significant association between Frailty score and FT4, as well as the presence of an inverse, statistically significant relationship between Frailty score and FT3 ( $r=-0.436 ; P<0.001$ ). In Figure 1B, it is evident how the two groups, namely, fractured and control, are clearly distinct from each other. An ANCOVA showed that FT3 values, despite being strongly dependent on the fracture, remained closely associated with the degree of frailty of the subject (Figure 1C).

In the entire study population, FT3 was significantly lower in frail subjects compared to prefrail and not frail subjects (Figure 2) (ANOVA $F=6.64 ; P<0.005$ ).

Table I Characteristics of the study population

\begin{tabular}{|c|c|c|c|c|}
\hline Parameters & $\begin{array}{l}\text { Total population } \\
(n=|| 2)\end{array}$ & $\begin{array}{l}\text { Patients admitted } \\
\text { for fracture }(n=62)\end{array}$ & $\begin{array}{l}\text { Outpatients } \\
(n=50)\end{array}$ & $P$-value \\
\hline Age, years & $79.1 \pm 7.0$ & $79.9 \pm 7.7$ & $78.1 \pm 6.0$ & 0.1792 \\
\hline Body weight, kg & $68.68 \pm 14.29$ & $66.04 \pm 12.57$ & $71.58 \pm 15.58$ & 0.0486 \\
\hline Height, m & $1.62 \pm 0.09$ & $1.6 \mathrm{I} \pm 0.08$ & $1.63 \pm 0.09$ & 0.1526 \\
\hline $\mathrm{BMI}, \mathrm{kg} / \mathrm{m}^{2}$ & $26.12 \pm 5.06$ & $25.55 \pm 4.88$ & $26.75 \pm 5.23$ & 0.2324 \\
\hline Handgrip, kg & $20.39 \pm 8.48$ & $16.80 \pm 7.74$ & $23.98 \pm 7.69$ & $<0.0001$ \\
\hline Fat mass, $\%$ & $33.20 \pm 8.05$ & $34.60 \pm 8.36$ & $32.53 \pm 7.90$ & 0.3245 \\
\hline Fat mass, $\mathrm{kg}$ & $22.94 \pm 8.63$ & $21.87 \pm 8.15$ & $23.45 \pm 8.89$ & 0.4830 \\
\hline Lean body mass, kg & $43.60 \pm 9.34$ & $39.7 I \pm 6.66$ & $45.46 \pm 9.92$ & 0.0164 \\
\hline Bone mineral content, $\mathrm{kg}$ & $1.96 \pm 0.58$ & $1.69 \pm 0.47$ & $2.08 \pm 0.59$ & 0.0072 \\
\hline T-score for neck of femur & $-1.90 \pm 1.16$ & $-2.54 \pm 0.97$ & $-1.57 \pm 1.12$ & 0.0005 \\
\hline CIRS-G & $8.36 \pm 4.18$ & $8.0 \mathrm{I} \pm 4.32$ & $8.79 \pm 3.99$ & 0.3371 \\
\hline CIRS-Cl & $3.47 \pm 1.80$ & $3.20 \pm 1.79$ & $3.8 \mathrm{I} \pm \mathrm{I} .78$ & 0.0539 \\
\hline CIRS-S & $1.64 \pm 0.32$ & $1.62 \pm 0.33$ & $|.68 \pm 0.3|$ & 0.3304 \\
\hline $\mathrm{CCl}$ & $2.86 \pm 1.77$ & $2.80 \pm 1.80$ & $2.94 \pm 1.76$ & 0.6289 \\
\hline Frailty score & $2.01 \pm 1.84$ & $2.62 \pm 1.95$ & $1.30 \pm 1.43$ & 0.0002 \\
\hline MMSE & $25.57 \pm 4.00$ & $24.44 \pm 4.61$ & $26.6 I \pm 3.0 I$ & $0.006 \mathrm{I}$ \\
\hline$A D L$ & $5.30 \pm 1.30$ & $4.83 \pm 1.64$ & $5.82 \pm 0.39$ & 0.0012 \\
\hline IADL & $5.92 \pm 2.43$ & $5.02 \pm 2.71$ & $6.90 \pm \mathrm{I} .6 \mathrm{I}$ & 0.0009 \\
\hline MNA & $23.94 \pm 4.22$ & $22.74 \pm 4.74$ & $25.34 \pm 3.02$ & 0.0018 \\
\hline GDS & $9.44 \pm 8.35$ & $11.19 \pm 9.52$ & $7.47 \pm 6.33$ & 0.0759 \\
\hline
\end{tabular}

Note: Values are presented as mean \pm standard deviation.

Abbreviations: BMI, body mass index; CIRS-G, cumulative illness rating scale for geriatrics; $\mathrm{CIRS}-\mathrm{Cl}$, cumulative illness rating scale for geriatrics - comorbidity index; CIRS-S, cumulative illness rating scale for geriatrics - severity; CCl, Charlson comorbidity index; MMSE, Mini-Mental State Examination; ADL, activities of daily living; IADL, instrumental activities of daily living; MNA, Mini Nutritional Assessment; GDS, geriatric depression scale. 
Table 2 Laboratory parameters

\begin{tabular}{|c|c|c|c|c|}
\hline Parameters & $\begin{array}{l}\text { Total population } \\
(n=|l| 2)\end{array}$ & $\begin{array}{l}\text { Patients admitted } \\
\text { for fracture }(n=62)\end{array}$ & $\begin{array}{l}\text { Outpatients } \\
(n=50)\end{array}$ & $P$-value \\
\hline Hemoglobin, g/dL & $12.45 \pm 1.87$ & $11.65 \pm 1.80$ & $13.44 \pm 1.44$ & $<0.0001$ \\
\hline WBCs, $1000 / \mu \mathrm{L}$ & $8.09 \pm 2.92$ & $9.37 \pm 3.08$ & $6.52 \pm 1.68$ & $<0.000$ I \\
\hline Glycemia, mg/dL & $120.20 \pm 40.73$ & $124.39 \pm 43.52$ & II $4.98 \pm 36.73$ & 0.2300 \\
\hline Creatinine, mg/dL & $1.05 \pm 0.62$ & $1.12 \pm 0.76$ & $0.97 \pm 0.36$ & 0.2038 \\
\hline Albumin, $g / d L$ & $3.35 \pm 0.79$ & $2.72 \pm 0.37$ & $4.12 \pm 0.35$ & $<0.0001$ \\
\hline Cortisol, $\mu g / d L$ & $17.73 \pm 6.83$ & $20.26 \pm 7.29$ & $14.59 \pm 4.62$ & $<0.000$ I \\
\hline hs-CRP, mg/dL & $5.92 \pm 6.64$ & $10.4 \pm 5.93$ & $0.45 \pm 0.64$ & $<0.0001$ \\
\hline IL-6, pg/mL & $64.94 \pm 74.70$ & $109.89 \pm 76.73$ & $|3.73 \pm| 7.9 \mid$ & $<0.0001$ \\
\hline TNF- $\alpha, p g / m L$ & $11.17 \pm 9.38$ & $13.99 \pm 11.33$ & $7.69 \pm 4.22$ & 0.0008 \\
\hline $\mathrm{TSH}, \mu \mathrm{IU} / \mathrm{mL}$ & $2.05 \pm 2.04$ & $2.09 \pm 1.64$ & $2.0 \mathrm{I} \pm 2.47$ & 0.8476 \\
\hline $\mathrm{FT} 3, \mathrm{pg} / \mathrm{mL}$ & $2.54 \pm 0.7 \mathrm{I}$ & $2.02 \pm 0.47$ & $3.15 \pm 0.38$ & $<0.000$ I \\
\hline $\mathrm{FT} 4, \mathrm{ng} / \mathrm{dL}$ & $1.24 \pm 0.20$ & $1.23 \pm 0.21$ & $1.25 \pm 0.21$ & 0.5682 \\
\hline
\end{tabular}

Note: Values are presented as mean \pm standard deviation.

Abbreviations: WBC, white blood cell; hs-CRP, high-sensitivity C-reactive protein; IL, interleukin; TNF, tumor necrosis factor; TSH, thyroid stimulating hormone; FT3, free triiodothyronine; FT4, free thyroxine.

In a logistic regression model in which frailty was considered the dependent variable, the best predictor of the hormonal parameters of frailty was represented by FT3 $\left(\chi^{2}=4.358 ; P<0.05\right)$.

Analysis of the ROC curve (Figure 3) showed that, in our study population, FT3 is an accurate parameter for identifying vulnerable subjects (AUC $=0.8520 ; P<0.001$ ). Taking FT3 values less than $2.3 \mathrm{pg} / \mathrm{mL}$ as cutoff, FT3 is able to distinguish frail subjects from the not frail ones, with a sensitivity of $73.91 \%$ ( $95 \%$ confidence interval [CI]: $51.60 \%-89.77 \%)$ and a specificity equal to $74.19 \%$ (95\% CI: $55.39 \%-88.14 \%$ ).

\section{Discussion}

Patients with hip fractures had a significant reduction in serum concentrations of FT3, but not of FT4. These changes are suggestive of the presence of NTIS. It is well known that NTIS is a clinical condition characterized by changes in the serum concentrations of thyroid hormones not caused by intrinsic abnormalities of thyroid function. ${ }^{21}$ Often, markedly

Table 3 FT3 and FT4 correlations

\begin{tabular}{llllll}
\hline Scales & FT3 & & & FT4 & \\
\cline { 2 - 3 } \cline { 5 - 6 } & $\boldsymbol{r}$ & $\boldsymbol{P}$-value & & $\boldsymbol{r}$ & $\boldsymbol{P}$-value \\
\hline MNA $^{\#}$ & $0.40 \mathrm{I}$ & 0.0001 & & 0.029 & 0.7849 \\
ADL $^{*}$ & 0.339 & 0.0012 & & 0.202 & 0.0579 \\
IADL* & 0.344 & 0.0009 & & 0.193 & 0.0697 \\
CIRS-G* & -0.210 & 0.0412 & & 0.017 & 0.8672 \\
Handgrip, kg & 0.354 & 0.0013 & & -0.002 & 0.9985 \\
\hline
\end{tabular}

Notes: \#Pearson's linear correlation. *Spearman's rank correlation.

Abbreviations: FT3, free triiodothyronine; FT4, free thyroxine; MNA, Mini Nutritional Assessment; ADL, activities of daily living; IADL, instrumental activities of daily living; CIRS-G, cumulative illness rating scale for geriatrics. reduced FT3 is observed in elderly subjects suffering from acute diseases or undergoing surgery. However, a slight reduction of FT3 values can also be found in apparently healthy elderly subjects. ${ }^{22,23}$

The prevalence of NTIS is high in the elderly, reaching $31.9 \%$ in elderly patients hospitalized for acute illness. ${ }^{24,25}$ The main alteration found in NTIS is the reduction of serum concentrations of either $\mathrm{T} 3$ or FT $3,{ }^{26}$ generally in association with normal or reduced concentrations of T4 or FT4, normal levels of TSH and increased levels of reverse T3 (rT3). ${ }^{26-28}$ From a pathogenic point of view, both the reduction of FT3 concentrations as well as the increase of rT3 concentrations appear to be the consequence of a reduction in the activity of type I deiodinase, an enzyme that physiologically promotes the conversion of $\mathrm{T} 4$ into $\mathrm{T} 3$ and the catabolism of rT3 into T2..$^{21,23}$

In the presence of acute or chronic diseases, as well as after surgery, an inflammatory process is activated, characterized by an increase in plasma concentrations of cytokines, such as IL-1, IL-6 and TNF- $\alpha$, apart from cortisol. ${ }^{29}$ Both the excess cortisol and cytokines appear to play an essential role in the pathogenesis of NTIS. As reported in numerous studies in humans, high levels of glucocorticoids suppress the pituitary response to thyrotropin releasing hormone (TRH), resulting in a reduction in serum concentrations of TSH and causing a modest decline in the levels of thyroid hormones. ${ }^{30}$ Meanwhile, IL-6 appears to have a direct inhibitory role on the activity of type I deiodinase, ${ }^{31-33}$ preventing the conversion of T4 into T3 and the catabolism of rT3.

In our study, the reduction in serum concentrations of FT3 is a clear manifestation of stress associated with a "fracture" event. The effect of a fracture on FT3 concentrations, 
A B

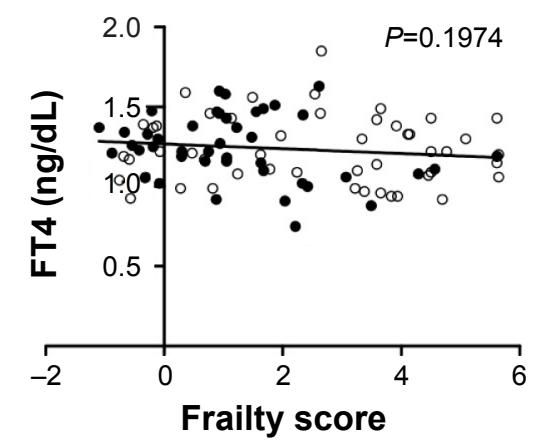

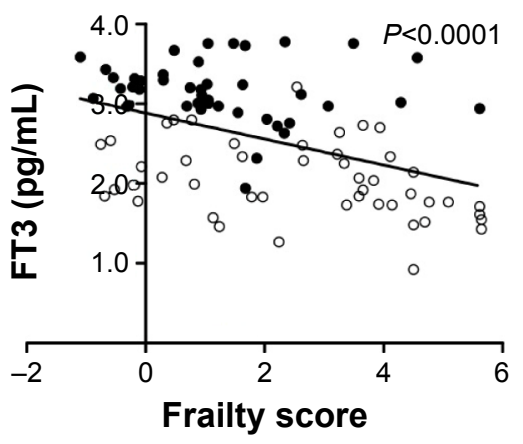

C

ANCOVA

Frailty score; $P=0.0084$

Patients with fractures; $P<0.0001$

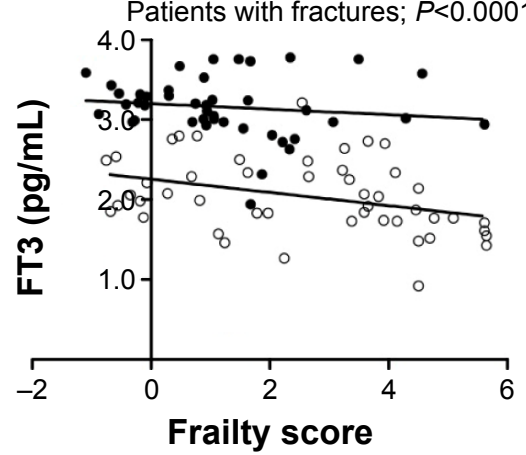

○ Patients with fractures $\bullet$ Controls

Figure I Linear correlation between FT4 and Frailty score (A); linear correlation between FT3 and Frailty score (B); effect of fracture on linear correlation between FT4 and Frailty score $(\mathbf{C})$.

Abbreviations: ANCOVA, analysis of covariance; FT3, free triiodothyronine; FT4, free thyroxine.

however, does not appear to be related only to the action of IL-6, other proinflammatory cytokines and hormones responding to stress, such as cortisol, although their concentrations significantly increase in fracture patients compared with the levels in control subjects. Numerous preexisting factors, such as the fracture patient's nutritional status, sarcopenia, disability and comorbidities, which characterize the condition of frailty and influence its pathogenesis, are strongly correlated with the values of FT3.

Several studies have shown the existence of a significant relationship between frailty, disability and comorbidities ${ }^{3}$ and have established that, although frailty is a condition distinct from comorbidity and disability, it often overlaps with the latter two: when associated together, frailty and comorbidities can predict disability, disability can induce frailty and worsen comorbidity, and comorbidity may contribute to the

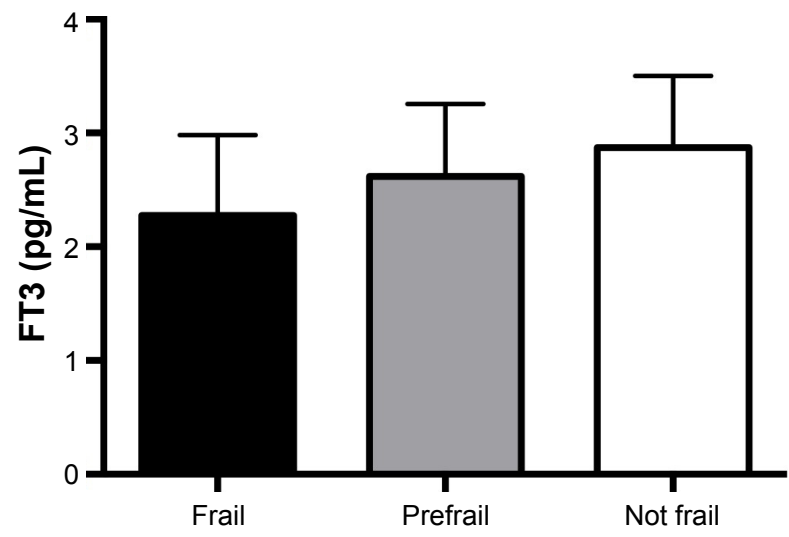

Figure 2 FT3 concentration (mean \pm SD) in frail, prefrail and not frail subjects. Note: ANOVA $F=6.64 ; P<0.005$.

Abbreviations: ANOVA, analysis of variance; FT3, free triiodothyronine; SD, standard deviation. development of frailty. ${ }^{34}$ These relationships, along with the direct inverse association between frailty and nutritional status, are well documented in several articles ${ }^{35-37}$ and were confirmed in our study (data not shown).

It is known that sarcopenia, defined as a syndrome characterized by a progressive loss of muscle mass and muscle strength, ${ }^{38}$ contributes to the development of frailty. ${ }^{39}$ A bone-muscle cross talk is involved in maintaining bone and muscle integrity. In sarcopenic patients with hip fragility fractures, reduced expression of bone morphogenetic proteins (BMP2-4) has been demonstrated. ${ }^{40}$

Our study also found that sarcopenia is inversely correlated with the Frailty score (data not shown). Similarly to the results obtained for the variable "frailty" regardless of the event of fracture, in our study population, we observed lower values of FT3 in patients with a greater number and

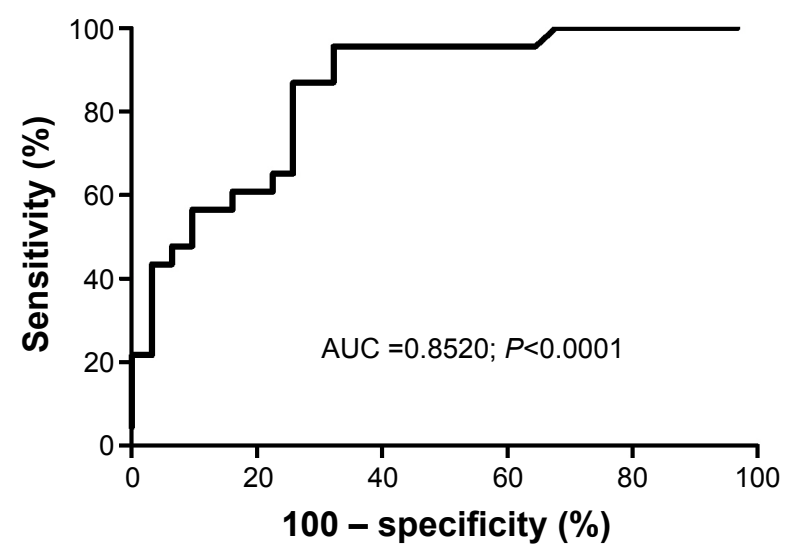

Figure 3 ROC curve for low FT3 concentration in the process of discriminating frail/prefrail subjects from not frail subjects.

Abbreviations: AUC, area under the curve; FT3, free triiodothyronine; ROC, receiver operating characteristic. 
greater severity of comorbidity, as well as in those with a worse nutritional status and with greater disability. Therefore, on the one hand, a reduction in FT3 depends on the same factors that contribute to frailty, which, in turn, may be worsened by low values of FT3 or may simply coexist with them; on the other hand, low values of FT3 can contribute to the development of frailty.

The hypothesis of a link between thyroid hormone abnormalities and frailty, which was recently suggested by Abdel-Rahman et $\mathrm{al}^{11}$ in patients with chronic kidney disease, is consistent with our data.

\section{Conclusion}

A reduction in FT3 may be considered to be the consequence of multiple events, such as malnutrition as well as acute and chronic diseases. The latter also contribute to determining frailty and are independently present before the event of a fracture, which, in turn, is a further important cause of a decline in FT3. Furthermore, as mortality is higher in frail subjects than in more robust subjects, ${ }^{3,41}$ several studies have shown that lower FT3 values are predictive of mortality. ${ }^{42}$

The diagnosis of NTIS is not univocal: as the dosage of rT3 is not available in clinical practice, it is generally linked to overcoming the threshold corresponding to the lower limit of normal FT3 levels. ${ }^{43}$ Both the increase in rT3 and a decline in FT3 are proportional to the severity of the syndrome and thus to the severity of the clinical condition. A decline in T3 is a progressive and continuous phenomenon that follows the increase in rT3, and it is therefore likely that the initial or milder forms of NTIS are not diagnosed solely on the basis of FT3 values.

We conclude that measuring FT3 can be a useful laboratory parameter in clinical assessment, which can play an important role in identifying vulnerable elderly subjects and in quantifying the condition of frailty. Furthermore, it deserves to be considered as an additional risk factor for mortality in elderly patients hospitalized for hip fracture.

\section{Study limitations}

There are two main limitations to the study: 1) the small sample of participants; 2) a possible selection bias in that many patients with fractures were cognitively compromised and thus not in a position to give informed consent for their participation in the study. Nevertheless, this limitation should have had such an effect as to reduce the differences observed.

\section{Acknowledgment}

The authors did not have any financial support for this study.

\section{Author contributions}

$\mathrm{AB}$ and $\mathrm{AV}$ participated in study concept and design, acquisition of subjects and data, analysis and interpretation of data, and manuscript preparation. MAC participated in acquisition of subjects and data. EG participated in acquisition of subjects and clinical management of patients with fractures. MF participated in study concept and design, acquisition of subjects, and data analysis. UT participated in study concept and design, as well as acquisition of subjects. All authors contributed toward data analysis, drafting and critically revising the paper and agree to be accountable for all aspects of the work.

\section{Disclosure}

The authors report no conflicts of interest in this work.

\section{References}

1. Christensen K, Doblhammer G, Rau R, Vaupel JW. Ageing populations: the challenges ahead. Lancet. 2009;374(9696):1196-1208.

2. ISTAT [homepage on the Internet]. Previsioni della popolazione. [Population Projection]. Available from: www.demo.istat.it. Accessed December 1, 2016.

3. Fried LP, Tangen CN, Walston J, et al. Frailty in older adults: evidence for a phenotype. J Gerontol A Biol Sci Med Sci. 2001;56(3):M146-M156.

4. Hogan DB, MacKnight C, Bergman H. Models, definitions, and criteria of frailty. Aging Clin Exp Res. 2003;15(3 suppl):1-29.

5. Rockwood K, Andrew M, Mitnitski A. A comparison of two approaches to measuring frailty in elderly people. J Gerontol A Bio Sci Med Sci. 2007;62(7):738-743.

6. Romero-Ortuno R, Walsh CD, Lawlor BA, Kelanny RA. A frailty instrument for primary care: findings from the Survey of health, ageing and retirement in Europe (SHARE). BMC Geriatr. 2010;10:57.

7. Howlett SE, Rockwood MRH, Mitnitski A, Rockwood K. Standard laboratory tests to identify older adults at increased risk of death. $B M C$ Med. 2014;12:171.

8. Roockwood K, McMillan M, Mitnitski A, Howlett SE. A frailty index based on common laboratory tests in comparison with a clinical frailty index for older adults in long-term care facilities. J Am Med Dir Assoc. 2015;16(10):842-847.

9. Morley JE. Hormones and the aging process. J Am Geriatr Soc. 2003; 51:S333-S337.

10. Morley JE, Malmstrom TK. Frailty, sarcopenia, and hormones. Endocrinol Metab Clin North Am. 2013;42(2):391-405.

11. Abdel-Rahman EM, Mansour W, Holley JL. Thyroid hormone abnormalities and frailty in elderly patients with chronic kidney disease: a hypothesis. Semin Dial. 2010;23(3):317-323.

12. Morley JE, Kim MJ, Haren MT. Frailty and hormones. Rev Endocr Metab Disord. 2005;6(2):101-108.

13. Fernández-Garrido J, Ruiz-Ros V, Buigues C, Navarro-Martinez R, Cauli O. Clinical features of prefrail older individuals and emerging peripheral biomarkers: a systematic review. Arch Gerontol Geriatr. 2014;59(1):7-17.

14. Katz S, Ford AB, Moskowitz RW, Jackson BA, Jaffe MW. Studies of illness in the aged. The index of ADL: a standardized measure of biological and psychosocial function. JAMA. 1963;185:914-919. 
15. Lawton MP, Brody EM. Assessment of older people: self-maintaining and instrumental activities of daily living. Gerontologist. 1969;9(3): 179-186.

16. Folstein MF, Folstein SE, McHugh PR. "Mini-mental state". A practical method for grading the cognitive state of patients for the clinician. J Psychiatr Res. 1975;12(3):189-198.

17. Yesavage JA, Brink TL, Rose TL, et al. Development and validation of a geriatric depression screening scale: a preliminary report. J Psychiatr Res. 1983;17(1):37-49.

18. Guigoz Y, Vellas B, Garry PJ. Assessing the nutritional status of the elderly: the Mini Nutritional Assessment as part of geriatric evaluation. Nutr Rev. 1996;54(1):S59-S65.

19. Parmelee PA, Thuras PD, Katz IR, Lawton MP. Validation of the Cumulative Illness Rating Scale in a geriatric residential population. J Am Geriatr Soc. 1995;43(2):130-137.

20. Charlson ME, Pompei P, Ales KL, MacKenzie CR. A new method of classifying prognostic comorbidity in longitudinal studies: development and validation. J Chronic Dis. 1987;40(5):373-383.

21. Warner MH, Beckett GJ. Mechanism behind the non-thyroidal illness syndrome: an update. J Endocrinol. 2010;205:1-13.

22. Nardi M, Di Bari M, Grasso L, et al. The "low-T3 syndrome" in unselected elderly home-dwellers: an epidemiological study in Dicomano, Italy. J Endocrinol Invest. 1999;22(10 suppl):40-41.

23. Mariotti S, Franceschi C, Cossarizza A, Pinchera A. The aging thyroid. Endocr Rev. 1995;16(6):686-715.

24. Boelaert K. Thyroid dysfunction in the elderly. Nat Rev Endocrinol. 2013;9(4):194-204.

25. Tognini S, Marchini F, Dardano A, et al. Non-thyroidal illness syndrome and short-term survival in a hospitalized older population. Age Aging. 2010;39:46-50.

26. Nicoloff JT. Thyroid function in nonthyroidal disease. In: De Groot LJ, editor. Endocrinology. 3rd ed. Philadelphia, PA: W. B. Saunders; 1995: 665-673.

27. Chopra IJ. Clinical review 86: euthyroid sick syndrome: is it a misnomer? J Clin Endocrinol Metab. 1997;82(2):329-334.

28. Van der Berghe G. Non-thyroidal illness in the ICU: a syndrome with different faces. Thyroid. 2014;24(10):1456-1465.

29. Boonen E, Van der Berghe G. Endocrine responses to critical illness: novel insights and therapeutic implications. J Clin Endocrinol Metab. 2014; 99(5):1569-1582.

30. De Groot LJ. Dangerous dogmas in medicine: the nonthyroidal illness syndrome. J Clin Endocrinol Metab. 1999;84(1):151-164.
31. Boelen A, Wiersinga WM, Koehrle J. Contributions of cytokines to nonthyroidal illness. Curr Opin Endocrinol Diabetes. 2006;13:444-450.

32. Wajner SM, Goemann IN, Bueno AL, Larsen PR, Maia AL. IL-6 promotes nonthyroidal illness syndrome by blocking thyroxine activation while promoting thyroid hormone inactivation in human cells. J Clin Invest. 2011;121(5):1834-1845.

33. Boelen A, Kwakkel J, Fliers E. Beyond low plasma T3: local thyroid hormone metabolism during inflammation and infection. Endocr Rev. 2011;32(5):670-693.

34. Fried LP, Ferrucci L, Darer J, Williamson JD, Anderson G. Untangling the concepts of disability, frailty, and comorbidity: implications for improved targeting and care. J Gerontol A Biol Sci Med Sci. 2004;59(3): 255-263.

35. Dorner TE, Luger E, Tschinderle J, et al. Association between nutritional status (MNA-SF) and frailty (SHARE-FI) in acute hospitalised elderly patients. J Nutr Healt Aging. 2014;18(3):264-269.

36. Bollwein J, Volkert D, Diekmann R, et al. Nutritional status according to the mini nutritional assessment (MNA) and frailty in community dwelling older persons: a close relationship. J Nutr Health Aging. 2013; 17(4):351-356.

37. Bonnefoy M, Berrut G, Lesourd B, et al. Frailty and nutrition: searching for evidence. J Nutr Health Aging. 2015;19(3):250-257.

38. Cruz-Jentoft AJ, Baeyens JP, Bauer JM, et al. Sarcopenia: European consensus on definition and diagnosis. Report of the European Working Group on Sarcopenia in Older People. Age Aging. 2010;39(4):412-423.

39. VanItallie TB. Frailty in the elderly: contributions of sarcopenia and visceral protein depletion. Metabolism. 2003;53(10):22-26.

40. Tarantino U, Scimeca M, Piccirilli E, et al. Sarcopenia: a histological and immunohistochemical study on age-related muscle impairment. Aging Clin Exp Res. 2015;27(suppl 1):S51-S60.

41. Cawthon PM, Marshall LM, Michael Y, et al; Osteoporotic Fractures in Men Research Group. Frailty in older men: prevalence, progression, and relationship with mortality. J Am Geriatr Soc. 2007;55(8): 1216-1223.

42. De Alfieri W, Nisticò F, Borgogni T, et al. Thyroid hormones as predictors of short- and long-term mortality in very old hospitalized patients. J Gerontol A Biol Sci Med Sci. 2013;68(9):1122-1128.

43. De Groot LJ [webpage on the Internet]. The non-thyroidal illness syndrome [updated February 1, 2015]. In: De Groot LJ, Beck-Peccoz P, Chrousos G, et al, editors. Endotext. South Dartmouth, MA: MDText. com, Inc.; 2000. Available from: http://www.ncbi.nlm.nih.gov/books/ NBK285570/. Accessed December 1, 2016.
Clinical Interventions in Aging

\section{Publish your work in this journal}

Clinical Interventions in Aging is an international, peer-reviewed journal focusing on evidence-based reports on the value or lack thereof of treatments intended to prevent or delay the onset of maladaptive correlates of aging in human beings. This journal is indexed on PubMed Central, MedLine,

\section{Dovepress}

CAS, Scopus and the Elsevier Bibliographic databases. The manuscript management system is completely online and includes a very quick and fair peer-review system, which is all easy to use. Visit http://www.dovepress. com/testimonials.php to read real quotes from published authors. 